\title{
The Interplay Between COVID-19 and Spondyloarthritis or Its Treatment
}

\author{
James T. Rosenbaum ${ }^{1}$ (D), Michael H. Weisman' ${ }^{2}$ D , Hedley Hamilton ${ }^{3}$, Cassie Shafer ${ }^{4}$, Elin Aslanyan ${ }^{4}$, \\ Richard A. Howard ${ }^{4}$, Kimberly Ogle ${ }^{6}$, John D. Reveille ${ }^{7}$ (D) Kevin L. Winthrop ${ }^{8}$ (D), \\ and Dongseok Choi ${ }^{8}$ iD
}

\begin{abstract}
Objective. The coronavirus disease 2019 (COVID-19) pandemic has created multiple uncertainties regarding rheumatic diseases or their treatment, with regard to the susceptibility to or severity of the viral disease. We aimed to address these questions as they relate to spondyloarthritis ( $\mathrm{SpA}$ ).

Methods. We created a longitudinal survey from April 10, 2020, to April 26, 2021. There were 4723 subjects with SpA and 450 household contacts who participated worldwide. Of these, 3064 respondents were from the US and $70.4 \%$ of them provided longitudinal data. To control for the duration of potential risk of COVID-19, the rate of contracting the disease was normalized for person-months of exposure.

Results. In an analysis of US subjects who provided longitudinal data, the incident rate ratio for the 159 (out of 2157) subjects who tested positive for COVID-19 was 1.16 compared to the US population as adjusted for age and sex (range $0.997-1.361, P=0.06$ ). A paired evaluation using patients and household members did not show a statistically significant effect to indicate a predisposition for developing COVID-19 as a result of SpA or its treatment. Our data failed to show that any class of medication commonly used to treat SpA significantly affected the risk of developing COVID-19 or increasing the severity of COVID-19.

Conclusion. These data do not exclude a small increased risk of developing COVID-19 as a result of SpA, but the risk, if it exists, is low and not consistently demonstrated. The data should provide reassurance to patients and to rheumatologists about the risk that COVID-19 poses to patients with SpA.
\end{abstract}

Key Indexing Terms: ankylosing spondylitis, axial spondyloarthritis, COVID, methotrexate, sulfasalazine, TNF inhibitor

The coronavirus disease 2019 (COVID-19) pandemic has been especially challenging for patients with inflammatory diseases, including spondyloarthritis ( $\mathrm{SpA})$. Although numerous studies have addressed how a COVID-19 infection affects patients with rheumatic diseases, ${ }^{1-16}$ the conclusions from these reports have not been consistent. Many, including our own, ${ }^{17}$ were performed early in the pandemic and as a consequence, they are often underpowered. In order to address the interplay between COVID-19 and $\mathrm{SpA}$ or its treatment, the Spondylitis Association of America (SAA), in collaboration with similar organizations around the world, conducted an online survey of patients with a diagnosis of $\mathrm{SpA}$ established by a physician. The survey began on April 10, 2020, and the data were locked as of April 26, 2021. We previously locked data on May 7, 2020, and reported our observations, but only 14 confirmed cases of COVID-19 were included at the time of report. ${ }^{17}$
This work was supported by the Spondylitis Association of America and Any-3. The Spondylitis Association of America receives support from AbbVie for this project. AbbVie played no role in the study design, data interpretation, or writing of the results. JTR receives support from the Grandmaison Fund for Autoimmunity Research, the William and Mary Bauman Foundation, the Stan and Madelle Rosenfeld Family Trust, and Research to Prevent Blindness.

IJ.T. Rosenbaum, MD, Departments of Medicine, Ophthalmology, and Cell Biology Oregon Health \& Science University, and Legacy Devers Eye Institute, Portland, Oregon, USA $;{ }^{2}$ M.H. Weisman, MD, Cedars Sinai Medical Center, Los Angeles, California, USA; ${ }^{3} \mathrm{H}$. Hamilton, $B S C$, Any-3, London, UK; ${ }^{4} C$. Shafer, BS, E. Aslanyan, BA, R.A. Howard, BA, Spondylitis Association of America, Los Angeles, California, USA; ${ }^{6} \mathrm{~K}$. Ogle, $B A$, Departments of Medicine, Ophthalmology, and Cell Biology, Oregon Health \& Science University, Portland, Oregon, USA; J.D. Reveille, MD, Department of Medicine, University of Texas, Houston, Texas, USA; ${ }^{8}$ K.L Winthrop, MD, MPH, D. Choi, PhD, OHSU-PSU School of Public Health and Departments of Medicine and Ophthalmology, Oregon Health \& Science University, Portland, Oregon, USA.
JTR consults for AbbVie, Gilead, UCB, Novartis, Roche, Horizon, Santen, Eyevensys, Corvus, Affibody, Revolo, Roivant, and Neoleukin; receives royalties from UpToDate; and receives grant support from Pfizer and Horizon. JTR serves on a data monitoring committee for Celgene (BMS). HH owns Any-3, the website that hosted the survey and donated its services. RAH owns stock in AbbVie, Amgen, BMS, GSK, Johnson and Johnson, Lilly, Merck, Novartis, Pfizer, and Teva. MHW consults for Novartis, UCB, Gilead, and GSK. JDR consults for UCB; and receives research support from Lilly and Janssen. KLW consults for Pfizer, AbbVie, UCB, Lilly, Galapagos, GSK, Roche, and Gilead; and receives research support from BMS and Pfizer. CS, EA, and RAH are employed by the Spondylitis Association of America. $D C$ and $K O$ report no conflicts of interest relevant to this article.

Address correspondence to Dr. J.T. Rosenbaum, Departments of Medicine, Ophthalmology, and Cell Biology, Oregon Health \& Science University, 3181 SW Sam Jackson Park Rd., L467Ad Portland, OR 97239, USA. Email:rosenbaj@obsu.edu. Accepted for publication September 23, 2021. 


\section{METHODS}

The survey instrument. The survey was reviewed and approved by the Oregon Health \& Science University Institutional Review Board (approval number 21375). Subjects were informed that by participating in this Web-based survey, informed consent was tacitly implied. Conclusions based on a data lock on May 7, 2020, have been previously reported. ${ }^{17}$ The survey was distributed to approximately 40,000 individuals (mostly in North America) who had registered with the SAA. In addition, the survey was distributed based on lists provided by the Axial Spondyloarthritis International Federation (ASIF). The survey was translated into 15 additional languages to accommodate ASIF members. This study complied with principles in the Declaration of Helsinki.

Modification of the Bath Ankylosing Spondylitis Disease Activity Index. The traditional Bath Ankylosing Spondylitis Disease Activity Index (BASDAI) includes 6 questions. The last 2 questions are both on morning stiffness. The responses to these last 2 questions are averaged and combined with the other 4 responses to create a single average. We reasoned that questions 5 and 6 are redundant and that the completion rate for the survey could be improved by combining these as a single question. In addition, as is true of many online surveys, we obtained responses as an integer from 1 to 10 , whereas the traditional BASDAI uses a continuous scale. Scores are based on the time of the initial response to the survey.

Statistical methods. Categorical variables were compared using chi-square testing with a Yates correction. Continuous variables were compared by $t$ test. Incident rate ratios (IRR) were compared using the Wald Test. To account for potential confounders in multiple regression analyses, Poisson regression model was also employed to estimate IRRs.

\section{RESULTS}

The present, updated survey results captured responses from 4723 subjects with $\mathrm{SpA}$ and 450 household contacts of these subjects. Moreover, 3289 subjects with SpA provided longitudinal data and 245 household contacts of 235 subjects with SpA also responded to the survey more than once. Of these, $64.9 \%$ of respondents were from the US and the rest were from 72 other countries, including $8 \%$ from Canada and $1.7 \%$ from the UK. The response rate from subjects with SpA living in North America was $9.3 \%$. Of the respondents, $63 \%$ were female. Males had a median age of 54 years and females responding to the survey had a median age of 49 years. We did not find that either age or sex had a statistically significant effect on the incidence of COVID-19, recognizing that the median age for our respondents was greater than the median age globally or for the US population. Of the respondents, $83.5 \%$ identified their disease as ankylosing spondylitis (AS). Arthritis with inflammatory bowel disease $(5.5 \%)$, psoriatic arthritis (PsA; 8.5\%), reactive arthritis (3.2\%), undifferentiated SpA (7.0\%), and nonradiographic SpA (7.0\%) were also chosen as appropriate diagnoses by respondents who were allowed to select $>1$ diagnostic category. Respondents who believed they had been exposed to COVID-19 constituted $19.6 \%$, and 384 (8.2\%) believed that they had been infected. Of those who believed they were infected, 295 had a confirmatory positive test. The conclusions that follow are based on those with confirmed disease.

To determine if $\mathrm{SpA}$ affects the likelihood of developing COVID-19, we focused on the analysis of respondents from the US who provided longitudinal data. We reasoned that a global analysis would suffer because of wide variation in incident rates in different countries. By analyzing only respondents who provided longitudinal data, we were able to calculate rates and avoid an enrollment bias common to most surveys (i.e., greater likelihood to participate if one is COVID-19-infected). Two thousand one hundred fifty-seven subjects were from the US and provided serial data. One hundred fifty-nine (7.4\%) of these subjects reported a positive test for COVID-19. Controlling for patient-months of exposure and comparing to the entire US population with adjustments for age and sex, we calculated an IRR of 1.16 (range $1.00-1.36, P=0.06$ ).

We analyzed our data on household contacts in 2 separate ways. Four hundred fifty household members from 434 households responded to our survey. Among the 16 households with $>1$ household member participating in the survey, we found 2 instances in which both household members were infected. For statistical purposes, we based the analysis on households and counted households with $>1$ reporting family member as a single household since reports were always concordant. Based on an analysis of 434 households, 11 controls developed COVID-19 and 14 subjects with SpA developed COVID-19. Thus, subjects with SpA were $27 \%$ more likely than controls to develop COVID-19, but the increase did not reach statistical significance by chi-square analysis with Yates correction $(P=0.61)$. Among the household controls, we had serial data on 245 subjects from 235 households. The incident rate of COVID-19 was calculated based on the number of subjects multiplied by the number of months of follow-up for household members or for subjects with SpA. The IRR was not statistically significant (IRR $1.15, P=0.74$ ). When we also analyzed only the US participants by using a Poisson regression model while accounting for age and sex, the estimated IRR was 1.14 $(P=0.81)$.

The small increase in COVID-19 incidence rate, if reproducible, could be due to multiple factors such as greater likelihood to be tested for COVID-19 because of SpA or increased susceptibility due to medications. The medications being taken at the time of the most recent survey are shown in Table 1 along with the BASDAI scores. The BASDAI scores were higher for all medicine groups compared to the no medication group. However,

Table 1. Relationship between the modified BASDAI and medication usage.

\begin{tabular}{lcccc}
\hline & $\mathrm{N}$ & $\begin{array}{c}\text { BASDAI, } \\
\text { Mean }\end{array}$ & SD & $P^{*}$ \\
\hline No SpA medication & 328 & 4.08 & 2.44 & NA \\
Sulfasalazine & 454 & 4.61 & 2.22 & 0.002 \\
Antimalarials & 152 & 5.33 & 2.17 & $<0.001$ \\
MTX & 568 & 5.26 & 2.19 & $<0.001$ \\
Anti-TNF & 2276 & 4.35 & 2.18 & 0.06 \\
Anti-IL-17 & 449 & 5.26 & 2.07 & $<0.001$ \\
Corticosteroid & 362 & 5.92 & 2.14 & $<0.001$ \\
JAK inhibitor & 71 & 5.70 & 2.04 & $<0.001$ \\
NSAID & 2525 & 4.84 & 2.11 & $<0.001$ \\
\hline
\end{tabular}

* Based on $t$ test against no SpA medication. BASDAI: Bath Ankylosing Spondylitis Disease Activity Index; IL: interleukin; JAK: Janus kinase; MTX: methotrexate; NA: not applicable; NSAID: nonsteroidal antiinflammatory drug; SpA: spondyloarthritis; TNF: tumor necrosis factor. 
it should be noted that BASDAI scores are often evaluated for change, and these scores are from a single point in time. Higher scores for those on specific medications presumably represent a confounding by indication (i.e., more aggressive treatment for more severe disease). The lowest scores were for those on no medication and the highest scores are for those taking a corticosteroid (CS) and/or a Janus kinase (JAK) inhibitor.

We next asked whether any class of medication influenced either the susceptibility to COVID-19 or the severity of the disease as judged subjectively by the respondent using a categorical scale from 1 to 10 (Table 2). The likelihood of developing COVID-19 is partially a function of time. A longer period of risk increases the likelihood that one will eventually develop the disease. Consequently, we dated the onset of the pandemic as March 2020, and normalized the data based on the number of months of potential exposure to the virus. Table 2 indicates that none of the treatments appeared to affect the likelihood of developing COVID-19 or the subjective rating of the severity of COVID-19. Some classes of medications such as antimalarials and JAK inhibitors were not commonly used by the respondents, so the statistical meaning of the results could be confounded by the limited size of the database.

One strength of our survey lies in its international distribution, but a weakness of the study is ironically the global nature, which introduces a great deal of heterogeneity for incidence rates. Accordingly, we repeated the analysis based on only the 3065 participants who were from the US. This analysis provided similar results (data not shown). Another strength of the study is its longitudinal nature, since this approach minimizes participation bias by enrolling patients prior to developing COVID-19. Of the subjects, $69.6 \%$ provided at least 1 follow-up response. Limiting the analysis only to those who provided follow-up again resulted in the same conclusions (data not shown). Finally, we restricted the analysis to the 2157 participants ( $70.4 \%$ of the US total) who were both from the US and provided follow-up data. Similar to the results shown in Table 1, US patients who provided follow-up consistently had a higher BASDAI if they were on medication $(P<0.001$ for each individual medication). As shown in Table 3, no medication had a statistically significant effect on the likelihood of contracting COVID-19 or the subjective severity of the infection.

Two recent studies ${ }^{18,19}$ have shown that COVID-19 replication in vitro is dependent on folate. Both studies suggested that methotrexate (MTX) might be useful as a treatment for COVID-19 infection. While combining MTX with other treatments such as remdesivir ${ }^{19}$ might prove valuable, our data did not find that MTX protected against developing COVID-19, nor was it associated with milder disease. CSs at higher dosages probably increase the risk of developing COVID-19. ${ }^{11}$ While we did not confirm this, our data are limited because we did not capture the dosage of CSs in the survey and CS usage is limited among patients with $\mathrm{SpA}$.

\section{DISCUSSION}

We are aware of only one other study that has used household members as a control for the amount of exposure to COVID-19. ${ }^{4}$ That study was based on 42 families and included 43 subjects with a rheumatic disease and 83 family members. Twenty-seven of the 43 subjects with rheumatic disease in Hubei, China, developed COVID-19 compared to only 28 of 83 family members. In the study from China, ${ }^{4}$ the subjects with rheumatic disease were 2.68 times as likely to contract COVID-19 based on an adjusted OR compared to household controls $(P<0.023)$. The study from Hubei explicitly excluded patients with AS based on the rationale that approximately only $10 \%$ of these subjects are treated with an anti-tumor necrosis factor.

The largest US study on COVID-19 and rheumatic diseases published at the time of our manuscript preparation was based on a review of electronic health records. ${ }^{9}$ It included 2379 subjects with rheumatic diseases and COVID-19, but only 76 of these subjects had AS. It concluded that rheumatic diseases are associated with greater severity of COVID-19. A recent study from Denmark on COVID-19 and rheumatic disease was much larger than our own, but it was only able to identify 7 patients with SpA who had been hospitalized due to COVID-19. ${ }^{16}$ The Global Rheumatology Alliance concluded that the use of sulfasalazine (SSZ) is associated with an increased risk of dying from COVID-19, comparable to the risk associated with rituximab

Table 2. Analysis of medication usage and the susceptibility and severity of COVID-19.

\begin{tabular}{lcccccccc}
\hline & $\begin{array}{c}\text { COVID-19 } \\
\text { Cases }\end{array}$ & $\begin{array}{c}\text { Person- } \\
\text { months }\end{array}$ & $\mathrm{RR}^{\mathrm{a}}$ & $95 \% \mathrm{CI}$ & $P^{*}$ & $\begin{array}{c}\text { Severity } \\
\text { Mean }\end{array}$ & $P^{* *}$ \\
\hline No SpA medication & 14 & 1937.7 & 1.00 & NA & NA & 4.14 & 2.25 & NA \\
MTX & 31 & 3671.4 & 1.17 & $0.62-2.20$ & 0.64 & 5.19 & 2.27 & 0.16 \\
HCQ & 5 & 941.0 & 0.74 & $0.26-2.04$ & 0.58 & 5.40 & 2.30 & 0.33 \\
Prednisone & 12 & 2271.9 & 0.73 & $0.34-1.58$ & 0.43 & 5.18 & 2.18 & 0.26 \\
Sulfasalazine & 31 & 2795.3 & 1.53 & $0.82-2.88$ & 0.18 & 4.45 & 2.20 & 0.67 \\
Anti-TNF & 135 & 14856.9 & 1.26 & $0.73-2.18$ & 0.42 & 4.75 & 2.40 \\
NSAID & 155 & 15708.7 & 1.37 & $0.79-2.36$ & 0.26 & 4.72 & 0.35 \\
Anti-IL-17 & 27 & 2985 & 1.25 & $0.66-2.39$ & 0.50 & 4.44 & 2.32 \\
JAK inhibitor & 6 & 439.7 & 1.89 & $0.73-4.91$ & 0.21 & 6.20 & 0.37 \\
\hline
\end{tabular}

${ }^{a} \mathrm{RR}$ is calculated relative to patients taking no medication. ${ }^{\mathrm{b}}$ Mean severity is the subjective severity of COVID-19 infection using a scale of 1 (mildest) to 10 (most severe). ${ }^{*}$ Wald test. ${ }^{* *} t$ test against no SpA medication. COVID-19: coronavirus disease 2019; HCQ: hydroxychloroquine; IL: interleukin; JAK: Janus kinase; MTX: methotrexate; NA: not applicable; NSAID: nonsteroidal antiinflammatory drug; RR: rate ratio; TNF: tumor necrosis factor. 
Table 3. Analysis of medication usage and susceptibility and severity of COVID-19 based on US participants who provided follow-up data.

\begin{tabular}{|c|c|c|c|c|c|c|c|c|}
\hline Medication (n) & $\begin{array}{c}\text { COVID-19 } \\
\text { Cases }\end{array}$ & $\begin{array}{l}\text { Person- } \\
\text { months }\end{array}$ & $\mathrm{RR}$ & $95 \% \mathrm{CI}$ & $P^{*}$ & $\begin{array}{c}\text { Severity, } \\
\text { Mean }\end{array}$ & SD & $P^{* *}$ \\
\hline No SpA medication (223) & 8 & 1349.7 & 1.00 & NA & NA & 4.13 & 2.36 & NA \\
\hline $\operatorname{MTX}(271)$ & 21 & 2479.2 & 1.43 & $0.63-3.23$ & 0.40 & 5.10 & 2.21 & 0.33 \\
\hline $\operatorname{HCQ}(81)$ & 4 & 759.4 & 0.89 & $0.27-2.95$ & 0.87 & 4.75 & 2.06 & 0.65 \\
\hline Prednisone (195) & 10 & 1722.6 & 0.98 & $0.39-2.48$ & 0.96 & 5.00 & 2.21 & 0.43 \\
\hline NSAID (1171) & 91 & 10698.7 & 1.44 & $0.70-2.96$ & 0.33 & 4.55 & 2.31 & 0.64 \\
\hline Anti-IL-17 (239) & 17 & 2115.6 & 1.36 & $0.59-3.14$ & 0.49 & 5.07 & 2.15 & 0.36 \\
\hline JAK inhibitor (41) & 5 & 361.7 & 2.33 & $0.76-7.13$ & 0.16 & 6.00 & 2.58 & 0.27 \\
\hline
\end{tabular}

${ }^{*}$ Wald test. ${ }^{* *} t$ test against no SpA medication. COVID-19: coronavirus disease 2019; HCQ: hydroxychloroquine; IL: interleukin; JAK: Janus kinase; MTX: methotrexate; NSAID: nonsteroidal antiinflammatory drug; RR: rate ratio; SpA: spondyloarthritis; TNF: tumor necrosis factor.

(RTX). ${ }^{11}$ As we recently reported, our data do not show increased likelihood to develop COVID-19 or to suffer from more severe COVID-19 in association with the use of SSZ..$^{20}$

We believe that our study adds to the growing literature on rheumatic disease and COVID-19 in several important ways. To our knowledge, it is by far the largest study to date on SpA and COVID-19. Studies on specific rheumatic diseases and COVID-19 are valuable. Just as a medication that benefits one rheumatic disease such as RTX for granulomatosis with polyangiitis might not benefit another rheumatic disease such as SpA, each underlying rheumatic disease could independently affect the response to a virus. Extrapolating from rheumatoid arthritis (RA) or systemic lupus erythematosus to $\mathrm{SpA}$ is inherently flawed. Recognizing that rates of COVID-19 infection vary widely based on date and geographic location, we analyzed specifically the subset of respondents who were both from the US and provided follow-up information. We found a $16 \%$ increase in the IRR with borderline statistical significance $(P=0.06)$. Our paired data using household controls account optimally for the likelihood of exposure to COVID-19. These data are arguably the best way to determine if SpA affects susceptibility to or severity of COVID-19. The longitudinal design is also innovative and reduces the inherent enrollment bias in survey studies. Statistics are ideal at showing differences, but they are less powerful at proving similarity. Our data do not show definitively that SpA does not affect susceptibility to COVID-19, since there is a small positive trend toward increased viral disease. However, this increase does not reach statistical significance. Additional studies or additional follow-up of our present study are required to answer the question with more confidence. The high $P$ value $(P=0.74)$ from our analysis based on household controls is reassuring in that if there is an effect, it is small. Further, if an effect exists, our data cannot exclude the possibility that medications rather than the disease itself account for the increase. Our conclusion regarding SpA and the severity of COVID-19 contrasts with the conclusions from at least 3 studies ${ }^{4,911}$ that included a range of rheumatic diseases rather than focusing on SpA.

All survey studies suffer from a selection bias as to who responds. However, our methodology allowed for the enrollment of subjects almost always prior to the development of infection with COVID-19, and we followed $70.4 \%$ of respondents longitudinally, with an extremely good response rate to repeated surveys after the first response. The longitudinal design reduces the bias from selective response to the survey. In addition, the inclusion of household controls allowed us to minimize confounding effects resulting from the vast range of environmental exposures. The survey nature of our study with protection of subject identity did not allow us to confirm diagnoses on the basis of a review of medical records, although we asked that participants have a diagnosis of SpA confirmed by a physician. We cannot exclude confounding because patients taking certain medications, such as a biologic, might exercise greater care to socially distance and minimize exposure. This same behavioral change could apply to those with SpA. We caution as well that our results should not be extrapolated to other rheumatic diseases, as some have comorbidities such as obesity with PsA and interstitial lung disease with RA that presumably affect susceptibility to and severity of coronaviral infection. Another limitation of our study is the inability to capture a fatal outcome from a COVID-19 infection. Similarly, subjects in an intensive care unit would be unlikely to respond to a survey. Finally, we recognize that emerging variants of COVID-19 might behave differently from the variants that predominated at the time of our survey.

Our data should reassure both patients and physicians that patients with SpA are not at a large increased risk for COVID-19 infection, nor does its treatment substantially add to this risk. Continued acquisition of longitudinal data is certainly warranted.

\section{ACKNOWLEDGMENT}

We are grateful to Donna Grims from the Spondylitis Association of America, and Emily Papaspyru, Adam Dale, and Ian Burton from Any-3 for help with downloading data from the survey.

\section{DATA AVAILABILITY}

Data for this study may be obtained by contacting Hedley Hamilton at Any-3.

\section{REFERENCES}

1. Moiseev S, Avdeev S, Brovko M, et al. Rheumatic diseases in intensive 
care unit patients with COVID-19. Ann Rheum Dis 2021;80:e16.

2. Santos CS, Morales CM, Alvarez ED, Castro CA, Robles AL, Sandoval TP. Determinants of COVID-19 disease severity in patients with underlying rheumatic disease. Clin Rheumatol 2020;39:2789-96.

3. Fredi M, Cavazzana I, Moschetti L, Andreoli L, Franceschini F, Brescia Rheumatology COVID-19 Study Group. COVID-19 in patients with rheumatic diseases in northern Italy: a single-centre observational and case-control study. Lancet Rheumatol 2020;2:e549-56.

4. Zhong J, Shen G, Yang H, et al. COVID-19 in patients with rheumatic disease in Hubei province, China: a multicentre retrospective observational study. Lancet Rheumatol 2020; 2:e557-64.

5. Sarzi-Puttini P, Marotto D, Caporali R, et al. Prevalence of COVID infections in a population of rheumatic patients from Lombardy and Marche treated with biological drugs or small molecules: a multicentre retrospective study. J Autoimmun 2021;116:102545.

6. Haberman RH, Castillo R, Chen A, et al; NYU WARCOV Investigators. COVID-19 in patients with inflammatory arthritis: a prospective study on the effects of comorbidities and disease-modifying antirheumatic drugs on clinical outcomes. Arthritis Rheumatol 2020;72:1981-9.

7. Favalli EG, Monti S, Ingegnoli F, Balduzzi S, Caporali R, Montecucco C. Incidence of COVID-19 in patients with rheumatic diseases treated with targeted immunosuppressive drugs: what can we learn from observational data? Arthritis Rheumatol 2020;72:1600-6.

8. Haberman R, Axelrad J, Chen A, et al. Covid-19 in immune-mediated inflammatory diseases - case series from New York. N Engl J Med 2020;383:85-8.

9. D'Silva KM, Jorge A, Cohen A, McCormick N, Zhang Y, Wallace ZS, et al. COVID-19 outcomes in patients with systemic autoimmune rheumatic diseases (SARDs) compared to the general population: a US multi-center comparative cohort study. Arthritis Rheumatol 2020;383:85-8.

10. Jorge A, D'Silva KM, Cohen A, et al. Temporal trends in severe COVID-19 outcomes in patients with rheumatic disease: a cohort study. Lancet Rheumatol 2021;3:e131-7.

11. Strangfeld A, Schafer M, Gianfrancesco MA, et al; COVID-19 Global Rheumatology Alliance. Factors associated with COVID-19-related death in people with rheumatic diseases: results from the COVID-19 Global Rheumatology Alliance physician-reported registry. Ann Rheum Dis 2021;80:930-42.

12. Kridin K, Schonmann Y, Solomon A, et al. Risk of COVID-19 infection, hospitalization, and mortality in patients with psoriasis treated by interleukin-17 inhibitors. J Dermatolog Treat 2021 March 24 (Epub ahead of print).

13. Bjornsson AH, Grondal G, Kristjansson M, Jonsdottir T, Love TJ, Gudbjornsson B; ICEBIO. Prevalence, admission rates and hypoxia due to COVID-19 in patients with rheumatic disorders treated with targeted synthetic or biologic disease modifying antirheumatic drugs or methotrexate: a nationwide study from Iceland. Ann Rheum Dis 2021;80:671-2.

14. Yousaf A, Gayam S, Feldman S, Zinn Z, Kolodney M. Clinical outcomes of COVID-19 in patients taking tumor necrosis factor inhibitors or methotrexate: a multicenter research network study. J Am Acad Dermatol 2021;84:70-5.

15. FAI2R/SFR/SNFMI/SOFREMIP/CRI/IMIDIATE consortium and contributors. Severity of COVID-19 and survival in patients with rheumatic and inflammatory diseases: data from the French RMD COVID-19 cohort of 694 patients. Ann Rheum Dis 2020;80:527-38.

16. Cordtz R, Lindhardsen J, Soussi BG, et al. Incidence and severeness of COVID-19 hospitalisation in patients with inflammatory rheumatic disease: a nationwide cohort study from Denmark. Rheumatology 2020 Dec 28 (Epub ahead of print).

17. Rosenbaum JT, Hamilton H, Choi D, Weisman MH, Reveille JD, Winthrop KL. Biologics, spondylitis and COVID-19. Ann Rheum Dis 2020;79:1663-5.

18. Zhang Y, Guo R, Kim SH, et al. SARS-CoV-2 hijacks folate and one-carbon metabolism for viral replication. Nat Commun 2021;12:1676.

19. Stegmann KM, Dickmanns A, Gerber S, et al. The folate antagonist methotexate diminishes replication of the coronavirus SARS-Cov-2 and enhances the antiviral efficacy of remdesivir in cell culture models. Virus Res 2021;302:198469.

20. Rosenbaum JT, Weisman MH, Shafer C, et al. Correspondence on 'Factors associated with COVID-19-related death in people with rheumatic diseases: results from the COVID-19 Global Rheumatology Alliance physician-reported registry'. Ann Rheum Dis 2021 May 13 (Epub ahead of print). 